\title{
Gesucht: ein Curriculum
}

An der achten Arbeitstagung zur nationalen Gesundheitspolitik wurde im Kongresszentrum am Messeplatz Basel über die «Stärkung der Grundversorgung» nachgedacht. Alle Gesundheitsberufe waren vertreten, eidgenössische und kantonale Behörden, Politiker, Versicherer, Soziologen und Fakultätsmitglieder. Im grossen Saal sprachen Bundesrat Couchepin und Regierungsräte, Gianfranco Domenighetti, Werner Bauer und Stefan Spycher, Leiter des Schweizerischen Gesundheitsobservatoriums (Obsan).

An drei Workshops wurden die Themen «Notfallkonzepte», «Welche Weiterbildung für welchen Hausarzt?» und «Hausarztmedizin der Zukunft» diskutiert, thesenhaft im Plenum präsentiert und von Pierre-Yves Maillard, Thomas Zeltner und Marc Müller kommentiert. Der Schweizerischen Gesundheitsdirektorenkonferenz (GDK) war es gelungen, mit kompetenten Referenten und einer hervorragenden Organisation einen beispielhaften Fachaustausch und Dialog zwischen unterschiedlichsten Akteuren zu vermitteln.

Wenn Altbewährtes erhalten werden soll, braucht es neue Wege im Medizinstudium, in der Weiterbildung und in der Forschung, aber auch neue Formen der Zusammenarbeit und neue Anreize für junge Ärztinnen und Ärzte, Allgemeinmediziner zu werden. Die gesellschaftlichen Bedingungen ändern sich rasant. Alle Zeichen deuten darauf hin, dass der freiberufliche Arzt in der Einzelpraxis ein Auslaufmodell ist. Es wird immer mehr Mütter-Hausärztinnen geben, die meistens in Teilzeit arbeiten, und zudem scheint gemäss Umfragen die nächste Generation Prestige und Geld weniger hoch einzuschätzen und dafür geregelte Arbeits- und Freizeitabläufe höher zu bewerten. Bisher spezifisch ärztliche Aufgaben werden an Nichtmediziner delegiert. Die Akademisierung der Pflegeberufe sorgt für Konkurrenz aus den Fachhochschulen, die auch für viele Spezialisten neue Arbeitsteilungen nach sich zieht. Alle Spitäler weiten ihre ambulanten Angebote, aus fachlichen und ökonomischen Gründen, zunehmend in die Grundversorgung aus. Eine Vorreiterrolle spielen dabei Privatspitäler, die mit Notfall- und Beratungsangeboten für neue Patienten werben oder für die weitere Nachbetreuung sorgen. Dabei spielen Callcenter in allen westlichen Ländern eine zunehmend wichtige, mit Versicherungsprämien finanzierte Rolle. Das Beispiel Holland zeigt, wie Notfalldienst und Grundversorgung durch Hausärzte konsequent auf der Basis einer regional organisierten Telefontriage neu ausgerichtet wurden. Die Bologna-Reform eröffnet neue Chancen, Studentinnen für die Hausarztmedizin zu motivieren. Allen scheint klar, dass der zukünftigen Praxisassistenz eine grosse Rolle zukommt, weil sich daraus die Praxisnachfolge rekrutieren dürfte. Der Kanton Waadt finanziert mit 200000 Franken zweimal sechs Monate, also vier Plätze jährlich. Gemäss GDK sollen die Kantone ähnliche Modelle übernehmen und dabei Lehrärzte unter Vertrag nehmen. Die Kantone und eventuell auch die Fakultäten und Fachgesellschaften können damit auf Praxismodelle Einfluss nehmen, die sie als vorbildlich und zukunftsgerichtet bevorzugen. Eine Vorstellung, die von den anwesenden Allgemeinpraktikern auch mit etlichem Unmut aufgenommen wurde. Unwidersprochen blieb die Forderung nach einem gut erkennbaren Berufsbild (Vision), nach Befriedigung und Anerkennung (Incentives) und einem ausgewogenen Verhältnis von Belastung und Erholung (Balance). Unbestritten war auch die Fachkompetenz, vage definiert als: breite Kenntnisse in Medizin, kontinuierliche inhaltliche Abstimmung mit Spezialisten und Fähigkeiten in nichtmedizinischen Bereichen. Wenig überraschend waren die auseinandergehenden Meinungen zu diesem «Schiff mit vielen Flaggen», das von der Bevölkerung sehr unterschiedlich wahrgenommen wird: als Hausarzt, immer präsent und gleich um die Ecke erreichbar, als Familienarzt, zuständig vom Säugling bis zur Grossmutter, mit Blick auf den sozialen Kontext, als Allgemeinarzt mit breitem Können für alles Fachliche und als Notfallarzt, kompetent und subito, vom Polytrauma bis zum verstauchten Fuss, vom Fieber bis zur Psychokrise.

Die Rede war auch von zukünftiger Forschung, vom gehäuften Burnout der Landärzte, von nachgewiesenen gesundheitlichen und ökonomischen Vorteilen der Hausarztmedizin, vom guten Angebot und von der entsprechenden Nachfrage in der Ostschweiz und dem entsprechenden West-Ost-Kostengefälle.

Metaphorisch tobte draussen der Jahrmarktrummel in der Abendsonne. Im Mittelpunkt der Power Tower, ein Freifallturm mit 13 Stockwerken.

Erhard Taverna 